\title{
A Survey on Face Detection and Recognition Techniques in Different Application Domain
}

\author{
Subrat Kumar Rath, Siddharth Swarup Rautaray \\ School of Computer Engineering, KIIT University, Bhubaneswar, Odisha, India \\ Email:subratrathm.tech@gmail.com, sr.rgpv@gmail.com
}

\begin{abstract}
In recent technology the popularity and demand of image processing is increasing due to its immense number of application in various fields. Most of these are related to biometric science like face recognitions, fingerprint recognition, iris scan, and speech recognition. Among them face detection is a very powerful tool for video surveillance, human computer interface, face recognition, and image database management. There are a different number of works on this subject. Face recognition is a rapidly evolving technology, which has been widely used in forensics such as criminal identification, secured access, and prison security. In this paper we had gone through different survey and technical papers of this field and list out the different techniques like Linear discriminant analysis, Viola and Jones classification and adaboost learning curvature analysis and discuss about their advantages and disadvantages also describe some of the detection and recognition algorithms, mention some application domain along with different challenges in this field.. We had proposed a classification of detection techniques and discuss all the recognition methods also. .
\end{abstract}

Index Terms - Leave Face detection, Feature extraction, face recognition.

\section{INTRODUCTION}

In recent years face recognition has received substantial attention from researchers in biometrics, pattern recognition, and computer vision communities. The machine learning and computer graphics communities are also increasingly involved in face recognition. Besides, there are a large number of commercial, securities, and forensic applications requiring the use of face recognition technologies. Face recognition has attracted much attention and its research has rapidly expanded by not only engineers but also neuroscientists, since it has many potential applications in computer vision communication and automatic access control system. Especially, face detection is an important part of face recognition as the first step of automatic face recognition[1]. However, face detection is not straightforward because it has lots of variations of image appearance, such as pose variation (front, nonfront), occlusion, image orientation, illuminating condition and facial expression. The aim of face detection is detect faces in any images or videos. Face detection can be regarded as a specific case of object-class detection. For the detection is to locate the face in the digital images/ video stream, no matter what the pose, scale, facial expression. In other words, face detection algorithms to handle pattern classification. It task to identify a given images to decides it has face or not. Face recognition has received significant attention in the last 15 years, due to the increasing number of commercial and law enforcement applications requiring reliable personal authentication (e.g. access control, surveillance of people in public places, security of transactions, mugs hot matching, and human-computer interaction) and the availability of low-cost recording devices.[1] Research in face recognition is motivated not only by the fundamental challenges this recognition problem poses but also by numerous practical applications where human identification is needed. Face recognition, as one of the primary biometric technologies which became more important owing to rapid advances in technologies such as digital cameras, the internet and mobile devices, and increased demands on security.

Further the paper is organized as follows: section two describes the application domain, section three focuses on background along with different face recognition algorithms and techniques, section four discuss about the discussion, finally the conclusion in the section five.

There are several challenges in face detection and recognition and they are as follows:

Illumination Challenged - Although the performance of face recognition systems in indoor platforms has reached a certain level, face recognition in outdoor platforms still remains as a challenging topic the effect of variation in the illumination conditions, which causes dramatic changes in the face appearance, is one of the most challenging problems that a practical face recognition system needs to achieve.

Face pose- In a surveillance system, the camera is mostly mounted to a location where the people cannot reach to the camera. Mounting a camera a high location, the faces are viewed by some angle degree. This is the simplest case in city surveillance applications. The next and the most difficult case is that people naturally pass through the camera view. They do not even look at the camera lens. Authorities cannot restrict people behaviours in public places. Recognition in such cases must be done in an accurate way. However, even state-of-the-art- 
techniques have 10 or 15degree angle limitation to recognize a face. Recognizing faces from more angles is another challenge.

Face expression-Face expression is less significant issue compare with angle and illumination but it affects the face.

Recognition results. Although a close eye or smiling face does affect the recognition rate by $1 \%$ to 10 percent, a face with large laugh has an influence as more as $30 \%$ since a laughing face changes the face appearance and distorts the correlation of eyes, mouth and nose.

Face aging-Face recognition algorithms are using either geometrical techniques or feature-based approaches or holistic methods. All of them do not solve the aging problem. Almost all of them give an age tolerance as long as 20 years after the training. Faces between 1 year and 15 years cannot be recognized since face appearance changes fast. Face appearance becomes stable after teenage years. A recognition algorithm that can recognize faces for all ages does not exist.

Dynamic Background- It is easier to recognise a face when the background is stable or single but problems arises when the background is moving or dynamic. Multiple face-Single face recognition easy in comparison to multiple face so it is also a big challenge in this field.

\section{APPLICATION DOMAIN}

There are numerous application areas in which FR can be exploited for these two purposes, a few of which are outlined below.

Verification (one-to-one matching): When presented with a face image of an unknown individual along with a claim of identity, ascertaining whether the individual is who he/she claims to be .[1]

Identification (one-to-many matching): Given an image of an unknown individual, determining that person's identity by comparing (possibly after encoding) that image with a database of (possibly encoded) images of known individuals.

Security-: access control to buildings, airports/seaports, ATM machines and border checkpoints; computer/ network security; email authentication on multimedia workstations.

Criminal justice systems-: Mug-shot/booking systems, post-event analysis, forensics.

Image database investigations-: Searching image databases of licensed drivers benefit recipients, missing children, immigrants and police bookings.

Smart Card applications-: In lieu of maintaining a database of facial images, the face-print can be stored in a smart card, bar code or magnetic stripe, authentication of which is performed by matching the live image and the stored template .

Access Control- Face verification, matching a face against a single enrolled exemplar, is well within the capabilities of current Personal Computer hardware. Since PC cameras have become widespread, their use for face-based PC logon has become feasible, though take-up seems to be very limited.[1]
Surveillance- The application domain where most interest in face recognition is being shown is probably surveillance. Video is the medium of choice for surveillance because of the richness and type of information that it contains and naturally, for applications that require identification, face recognition is the best biometric for video data.

Border Control -Biometrics technology is used to provide effective identification processes, and definitely a relevant security solution for Border Control/ Airports. Iris recognition, fingerprinting, document verification and vascular verification are all burgeoning Biometric technologies.[1]

\section{BACKGROUND}

It is the general opinion that advances in computer vision research will provide useful insights to neuroscientists and psychologists into how human brain works, and vice versa.. Face recognition is one of the most relevant applications of image analysis. It's a true challenge to build an automated system which equals human ability to recognize faces. Although humans are quite good identifying known faces, we are not very skilled when we must deal with a large amount of unknown faces. The computers, with an almost limitless memory and computational speed, should overcome humans limitations. The face recognition technique mainly work in three steps

1. Face detection 2. Feature extraction 3. Face recognition.

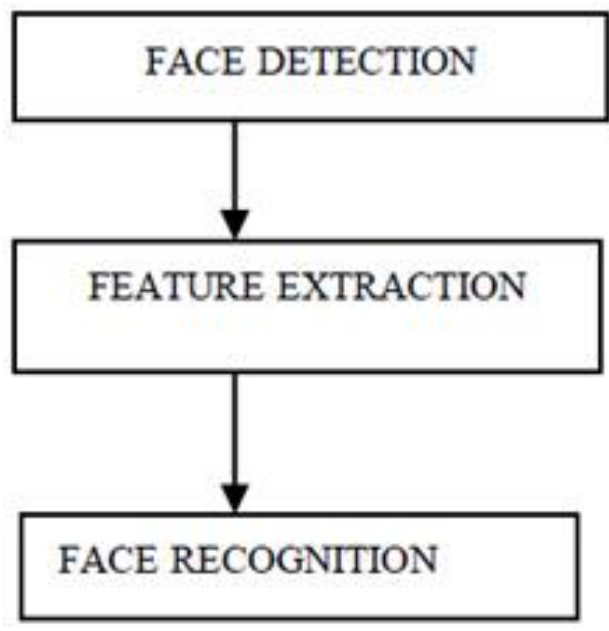

Fig. 1 Face detection and Recognition Overview

\subsection{FACE DETECTION}

Face detection can be regarded as a specific case of object-class detection. In object-class detection, the task is to find the locations and sizes of all objects in an image that belong to a given class. Face detection can be regarded as a more general case of face localization. In face localization, the task is to find the locations and sizes of a known number of faces (usually one). In face detection, one does not have this additional information. 


\subsection{FEATURE EXTRACTION}

The In pattern recognition and in image processing, feature extraction is a special form of dimensionality reduction. When the input data to an algorithm is too large to be processed and it is suspected to be notoriously redundant then the input data will be transformed into a reduced representation set of features . After the FD step, human-face patches are extracted from images. Directly using these patches for FR have some disadvantages, first, each patch usually contains over 1000 pixels, which are too large to build a robust recognition system. Second, face patches may be taken from different camera alignments, with different face expressions, illuminations, and may suffer from occlusion and clutter. To overcome these drawbacks, feature extractions are performed to do information packing, dimension reduction, salience extraction, and noise cleaning. After this step, a face patch is usually transformed into a vector with fixed dimension or a set of fiducial points and their corresponding locations. Transforming the input data into the set of features is called feature extraction.

\subsection{FACE RECOGNITION}

A facial recognition system is a computer application for automatically identifying or verifying a person from a digital image or a video frame from a video source. One of the ways to do this is by comparing selected facial features from the image and a facial database. It is typically used in security systems and can be compared to other biometrics such as fingerprint or eye iris recognition systems. Among the different biometric techniques, facial recognition may not be the most reliable and efficient. However, one key advantage is that it does not require aid (or consent) from the test subject. Properly designed systems installed in airports, multiplexes, and other public places can identify individuals among the crowd.

\subsection{ALGORITHM FOR FACE DETECTION AND FACE RECOGNITION}

\subsubsection{HAAR CLASSIFIER}

The core basis for Haar classifier object detection is the Haar-like features. These features, rather than using the intensity values of a pixel, use the change in contrast values between adjacent rectangular groups of pixels. The contrast variances between the pixel groups are used to determine relative light and dark areas. Two or three adjacent groups with a relative contrast variance form a Haar-like feature. Haar-like features are used to detect an image. Haar features can easily be scaled by increasing or decreasing the size of the pixel group being examined. This allows features to be used to detect objects of various sizes.

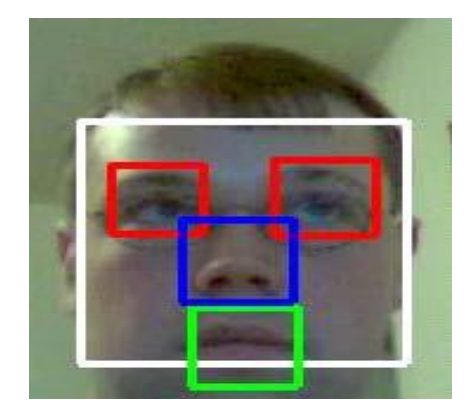

Fig. 2 Example of haar classifier detection

\subsection{2. $P C A$}

Derived from Karhunen-Loeve's transformation. Given an s-dimensional vector representation of each face in a training set of images, Principal Component Analysis (PCA) tends to find a t-dimensional subspace whose basis vectors correspond to the maximum variance direction in the original image space. This new subspace is normally lower dimensional $(\mathrm{t}<<\mathrm{s})$. If the image elements are considered as random variables, the PCA basis vectors are defined as eigenvectors of the scatter matrix.[2]

\subsubsection{ICA}

Independent Component Analysis (ICA) minimizes both second-order and higher-order dependencies in the input data and attempts to find the basis along which the data (when projected onto them) are - statistically independent. Bartlett et al. provided two architectures of ICA for face recognition task: Architecture I - statistically independent basis images, and Architecture II - factorial code representation.

\subsection{4. $L D A$}

Linear Discriminant Analysis (LDA) finds the vectors in the underlying space that best discriminate among classes. For all samples of all classes the between-class scatter matrix $S B$ and the within-class scatter matrix $S W$ are defined. The goal is to maximize $S B$ while minimizing $S W$, in other words, maximize the ratio $\operatorname{det}|S B| / \operatorname{det}|S W|$. This ratio is maximized when the column vectors of the projection matrix are the eigenvectors of $\left(S W^{\wedge}-1 \times S B\right)$.

\subsubsection{EP}

A eigen space-based adaptive approach that searches for the best set of projection axes in order to maximize a fitness function, measuring at the same time the classification accuracy and generalization ability of the system. Because the dimension of the solution space of this problem is too big, it is solved using a specific kind of genetic algorithm called Evolutionary Pursuit (EP).[2] 


\subsection{6. $E B G M$}

Elastic Bunch Graph Matching (EBGM). All human faces share a similar topological structure. Faces are represented as graphs, with nodes positioned at fiducially points. (exes, nose...) and edges labelled with 2-D distance vectors. Each node contains a set of 40 complex Gabor wavelet coefficients at different scales and orientations (phase, amplitude). They are called "jets". Recognition is based on labelled graphs. A labelled graph is a set of nodes connected by edges, nodes are labelled with jets, edges are labelled with distances.

\subsubsection{KERNEL METHODS}

The face manifold in subspace need not be linear. Kernel methods are a generalization of linear methods. Direct non-linear manifold schemes are explored to learn this non-linear manifold.

\subsubsection{TRACE TRANSFORM}

The Trace transform, a generalization of the Radon transform, is a new tool for image processing which can be used for recognizing objects under transformations, e.g. rotation, translation and scaling. To produce the Trace transform one computes a functional along tracing lines of an image. Different Trace transforms can be produced from an image using different trace functional.

\subsection{9. $A A M$}

An Active Appearance Model (AAM) is an integrated statistical model which combines a model of shape variation with a model of the appearance variations in a shape-normalized frame. An AAM contains a statistical model if the shape and gray-level appearance of the object of interest which can generalize to almost any valid example. Matching to an image involves finding model parameters which minimize the difference between the image and a synthesized model example projected into the image.[2]

\subsubsection{SVM}

Given a set of points belonging to two classes, a Support Vector Machine (SVM) finds the hyper plane that separates the largest possible fraction of points of the same class on the same side, while maximizing the distance from either class to the hyper plane. PCA is first used to extract features of face images and then discrimination functions between each pair of images are learned by SVMs.

\subsection{FACE DETECTION}

The Face detection has been one of the most studied topics in the computer vision literature. In this paper, This paper is about the recent advances in face detection for the past decade. This survey is about the various techniques according to how they extract features and what learning algorithms are adopted and after studied those methods we have divided them into different categories .we will discuss them one by one. The total face detection approach works through some algorithms, but here only HAAR CLASSIFIERS based method will discussed.[3]

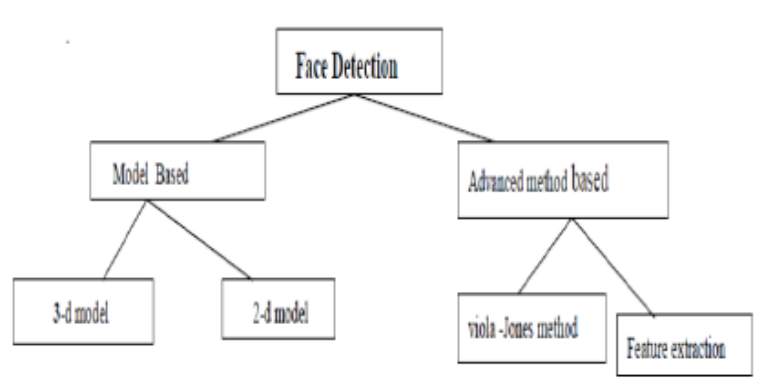

Fig. 3 face detection tree

The total detection method is divided in to two types

1.Model based based

2. Advanced method based

\subsubsection{MODEL BASED BASED}

In this section we placed the methods which detects the faces according to models means 2-d or 3-d model based. some other methods which are related to face detection and process the steps according to models we had placed them in the list.

\subsubsection{ADVANCED METHOD BASED}

In this section we had placed some advance methods and techniques which are based on some other methods

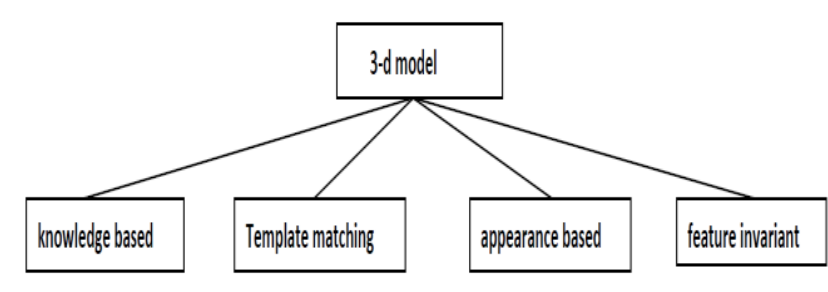

Fig. 3 (a) Face detection tree

\subsubsection{3-D MODEL BASED}

There is a growing demand for better facial recognition systems, those which have lesser or no problems with lightning, different angles and expressions. 3D facial recognition is an upcoming market, the techniques are getting better, the research completer and the hardware less expensive.

Now we will discuss the techniques which are falls under 3-D model

Knowledge-based methods -This model based on human knowledge of the typical human face geometry and facial features arrangement A hierarchical approach may be used, which examines the face at different resolution levels.[4]. 
Feature invariant Method -This aim to find structural features that exist even when the viewpoint or lighting conditions vary and then use these to locate faces. Different structural features are being used: facial local features, texture, and shape and skin color.[4]

Template Matching Approaches-To detect a face in a new image, first the head outline, which is fairly consistently roughly elliptical, is detected using filters, edge detectors, or silhouettes. Then the contours of local facial features are extracted in the same way, exploiting knowledge of face and feature geometry.

Appearance-based methods -While template matching methods rely on a predefined template or model, appearance-based methods use large numbers of examples (images of faces and $\backslash$ or facial features)depicting different variations (face shape, skin color, eye color, openlclosed mouth, etc). Face detection

can be viewed as a pattern classification problem with two classes: "face" and "non-face". The "nonface" class contains images that may depict anything that is not a face, while the "face" class contains all face images. [4]

\subsubsection{2-D-MODEL}

Video security systems are a well-known concept in daily life, nowadays 2D systems are the systems that are used.

Now we are listing out the techniques which falls under 2-d model.

2-d model is mainly divided in to two types

1. feature based

2. view based or image based

There are some other techniques fall under these two techniques we are listing them differently.

Feature based -The techniques under this method are

Low level analysis - This technique is based on the concept of analyzing low level visual features by using pixel properties like intensity levels, edges, and color properties.

Edge based face detection- Edge is the most primitive feature in computer vision applications and it was applied in some earlier face detection techniques by Sakai et al.. It was based on analyzing line drawings of faces to locate facial features.

Skin color based face detection-Skin color plays an important role in detecting faces in color images because skin chromaticity values of different color space can be effectively used to segment the input image. It helps to identify the probable regions containing faces.

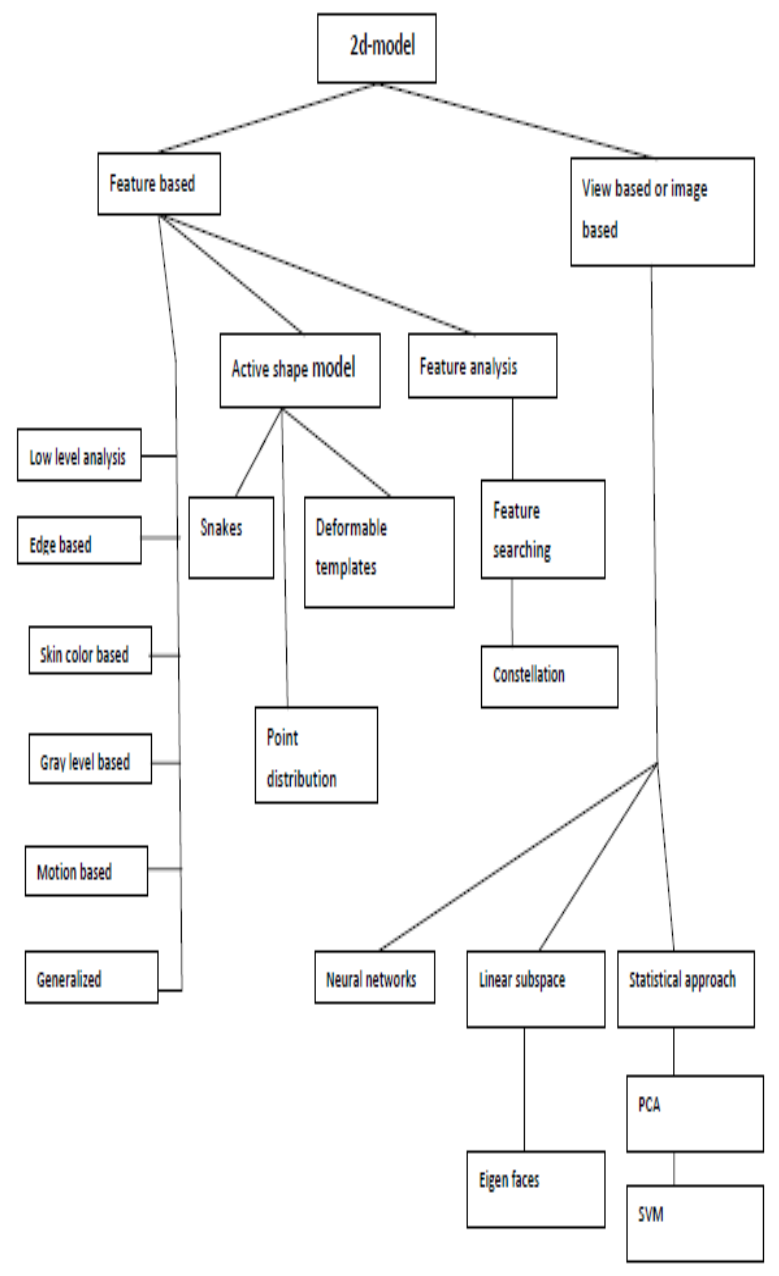

Fig. 3 (b) Face detection tree

Level based face detection-The gray information of an image can also considered as features. For example, facial features like eyebrows, pupils, and lips are usually darker than their surrounding regions. This property can be useful to differentiate various facial parts. Several recent facial feature extraction algorithms are basically search for local gray minima within segmented facial regions.

Motion based face detection- When use of video sequence is available, motion information can be used to locate moving objects. Moving silhouettes like face and body parts can be extracted by simply thresholding accumulated frame differences. Besides face regions, facial feature scan be located by frame differences.

Generalized measures -So far we have considered low level features like edges, skin color, gray level intensity and motion; all of these techniques are derived in the early stage of the human visual system. This visual system is nothing but the various responses made by our inner retina. This pre-attentive processing allows visual information to be organized in various bases prior to high-level visual activities in the brain.

Active Shape Models-Active shape models focus on complex non-rigid features like actual physical and higher level appearance of features. Active shape models use local features (edges, brightness) to find shape of 
feature models. Active shape models are divided into three groups: These are snakes, deformable templates, and point distribution models

Snakes -In this approach, active contours or snakes are used to locate head boundary. Also features' boundaries can be found by these contours. To achieve our task we have to initialize the starting position of the snake, which may be at the proximity around the head boundary.

Deformable Templates -Locating facial features' boundaries by using active contours is not easy task. Finding and locating facial edges is difficult. Sometimes there can be edge detection problems because of bad lighting or bad contrast of image. So, we need more flexible methods. Deformable templates approaches are developed to solve this problem. Deformation is based on local valley, edge, peak, and brightness. Other than face boundary, salient feature (eyes, nose, mouth and eyebrows) extraction is a great challenge of face recognition. In this method some predefined templates are used to guide the detection process. These predefined templates are very flexible and able to change their size and other parameter values to match themselves to the data. The final values of these parameters can be used to describe the features.

Point Distribution Models -These models are compact parameterized descriptions of the shapes based on statistics. The implementation process of PDM is quite different from the other active shape models. The contour of PDM is discretized into a set of labeled points. Now, the variations of these points can be parameterized over a training set that that includes objects of different sizes and poses. We can construct these variations of features as a linear flexible model.

There are some techniques are which falls under this

Neural Network based face detection -In Rowley's (1999) research a view based approach to detect faces in still images was introduced, and proved that face detection problem can be effectively solved by using Neural Networks to detect frontal, and non-frontal faces with different

poses and rotation degree. But the problem when we use a machine learning technique to learn detecting faces was that faces in images vary considerably with different lighting conditions, pose, occlusion and facial expressions, compensating for these variations was important for the learning process.

Neural networks can be applied successfully in face detection systems. The advantage of using neural networks for face detection is the feasibility of training a system to capture the complex class conditional density of face images.

Constellation Analysis-All those approaches discussed so far are rigid in nature; hence fail to solve some problem like locating faces of various poses in complex background. To overcome this problem later researches make a group of facial features in face-like constellations using more robust modeling methods such as statistical analysis.

Linear Subspace Methods -Human face images lie in a subspace of overall image space. By using this subspace concept, several analysis methods are developed. In image processing world, the most important three methods are principal component analysis (PCA), linear discriminant analysis, and factor analysis (FA).

Eigen faces. -In information theory concept, if we want to extract some information from a face image, we first encode it and then compare it with some other encoded face image on a database. A simple way to extract the information from a face image is to capture the variation in a collection of face images and use this information to encode and compare individual face images. Mathematically, we wish to find the principal components of distribution of faces or the eigenvectors of the covariance matrix of a set of face images. These eigenvectors are a set of features, which together characterize the variation between face images. Each image location contributes more or less to each eigenvectors, so that we can display the eigenvectors as a sort of ghostly face, which we call an eigenfeces. In the training set, each face image is represented by a linear combination of the eigenfaces. Hence the number of possible Eigen faces is same as the number of face images.

Statistical Approaches-Apart from linear subspace methods and neural networks, there are several other statistical approaches to face detection like systems based on information theory, a support vector machine, and Bayes' decision rule.

Support Vector Machine (SVM)- According to P. Jonathon Phillips Support Vector Machines (SVMs) are formulated to solve a classical two-class pattern recognition problem. We adapt SVM to face recognition by modifying the interpretation of the output of a SVM classifier and devising a representation of facial images that is concordant with a two-class problem. Traditional SVM returns a binary value, the class of the object. To train our SVM algorithm, we formulate the problem in a difference space, which explicitly captures the dissimilarities between two facial images. This is a departure from traditional face space or view-based approaches, which encodes each facial image as a separate view of a face.

Principal Components Analysis (PCA) -PCA is a technique based on the concept of eigen faces and was first introduced by Kirby and Sirivich in 1988. PCA also known as Karhunen Loeve projection). It is one of the more successful techniques of face recognition and easy to understand and describe using mathematics. This method involves using Eigen faces. Eigen faces have been used to track human faces. They use a principal component analysis approach to store a set of known patterns in a compact subspace representation of the image space, where the Eigen vectors of the training image set span the subspace. In this method, the input image and images of the eigen face gallery should be the same size and we have to normalize the input image so that the eyes, nose and mouth are properly lined up i.e. we only consider the face region and that should be a frontal face. In case of a non-frontal face it may result poor performance.[5]. 


\subsection{ADVANCED METHOD BASED}

In this section we will discuss about different new methods and techniques .we divided this in to two type

1. Viola -Jones method, 2-Feature extraction

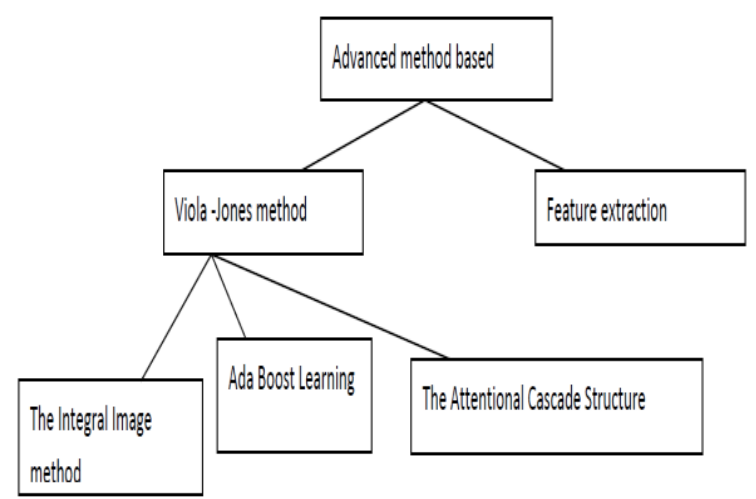

Fig. 3(c) Face detection tree

Viola -Jones method -If one were asked to name a single face detection algorithm that has the most impact in the 2000's, it will most likely be the seminal work by Viola and Jones. The Viola-Jones face detector contains three main ideas that make it possible to build a successful face detector that can run in real time: the integral image, classifier learning with AdaBoost, and the attentional cascade structure.[5]

The Integral Image-Integral image, also known as a summed area table, is an algorithm for quickly and efficiently computing the sum of values in a rectangle subset of a grid. It was first introduced to the computer graphics field by Crow for use in mip maps. Viola and Jones applied the integral image for rapid computation of Haar-like features.[5]

AdaBoost Learning-_Boosting is a method of finding a highly accurate hypothesis by combining many "weak" hypotheses, each with moderate accuracy.[5]

The Attentional Cascade Structure-Attentional cascade is a critical component in the Viola- Jones detector. The key insight is that smaller, and thus more efficient, boosted classifiers can be built which reject most of the negative sub-windows while keeping almost all the positive examples. Consequently, majority of the sub windows will be rejected in early stages of the detector, making the detection process extremely efficient.[5]

Feature Extraction-As mentioned earlier, thanks to the rapid expansion in storage and computation resources, appearance based methods have dominated the recent advances in face detection. The general practice is to collect a large set of face and no face examples, and adopt certain machine learning algorithms to learn a face model to perform classification. There are two key issues in this process: what features to extract, and which learning algorithm to apply.

\subsection{FACE RECOGNITION TECHNIQUES}

The method for acquiring face images depends upon the underlying application. For instance, surveillance applications may best be served by capturing face images by means of a video camera while image database investigations may require static intensity images taken by a standard camera. Therefore, depending on the face data acquisition methodology, face recognition techniques can be broadly divided into three categories: methods that operate on intensity images, those that deal with video sequences, and those that require other sensory data such as 3D information or infra-red imagery. The following discussion sheds some light on the methods in each category and attempts to give an idea of some of the benefits and drawbacks of the schemes mentioned there in general.

Recognizing From One Sample per Person -In this section, we review existing methods dealing with robust face recognition from a single intensity image. We have broadly classified these methods into three categories, according to the type of features used by various methods; some methods clearly overlap category boundaries and are discussed at the end of this section.

Local methods. These methods use the local facial features for recognition. Care should be taken when deciding how to incorporate global configurational information into local face model.

Hybrid methods. These methods use both the local and holistic features to recognize a face. These methods have the potential to offer better performance than individual holistic or local methods, since more comprehensive information could be utilized.

\subsubsection{LOCAL FEATURE-BASED METHODS}

Most of earlier face recognition methods belong to this category. In these methods, usually only a single image per person is used to extract geometrical measures such as the width of the head, the distances between the eyes, and so on. The extracted features are then stored in the database as templates for later matching usage. In early 1990s, Brunelli and Poggio described a face recognition system, which can automatically extract 35 geometrical features to form a 35-dimensional vector for face representation, and the similarity matching is performed with a Bayes classifier.

\subsubsection{HYBRID METHODS}

Hybrid methods are those approaches using both holistic and local features. The key factors that influence the performance of hybrid methods include how to determine which features should be combined and how to combine, so as to preserve their advantages and avert their disadvantages at the same time. These problems have close relationship with the multiple classifier system and ensemble learning in the field of machine learning. Unfortunately, even in these fields, these problems remain unsolved. In spite of this, numerous efforts made in these fields indeed provide us some insights into solving these problems, and these lessons can be used as guidelines in designing a hybrid face recognition system. 


\subsubsection{FACE RECOGNITION FROM INTENSITY IMAGES}

Face recognition methods for intensity images fall into two main categories: feature-based and holistic. An overview of some of the well-known methods in these categories is given below.

\subsubsection{FEATURED-BASED}

Feature-based approaches first process the input image to identify and extract (and measure) distinctive facial features such as the eyes, mouth, nose, etc., as well as other fiducial marks, and then compute the geometric relationships among those facial points, thus reducing the input facial image to a vector of geometric features. [6].

Another well-known feature-based approach is the elastic bunch graph matching method proposed by Wiskott. This technique is based on Dynamic Link Structures. A graph for an individual face is generated as follows: a set of fiducial points on the face are chosen. Each fiducial point is a node of a full connected graph, and is labeled with the Gabor filters' responses applied to a window around the fiducial point. Each arch is labeled with the distance between the correspondent fiducial points. A representative set of such graphs is combined into a stack-like structure, called a face bunch graph. Once the system has a face bunch graph, graphs for new face images can then be generated automatically by Elastic Bunch Graph Matching. Recognition of a new face image is performed by comparing its image graph to those of all the known face images and picking the one with the highest similarity value. Using this architecture, the recognition rate can reach $98 \%$ for the first rank and $99 \%$ for the first 10 ranks using a gallery of 250 individuals. The system has been enhanced to allow it to deal with different poses. but the recognition performance on faces of the same orientation remains the same. Though this method was among the best performing ones in the most recent FERET evaluation.
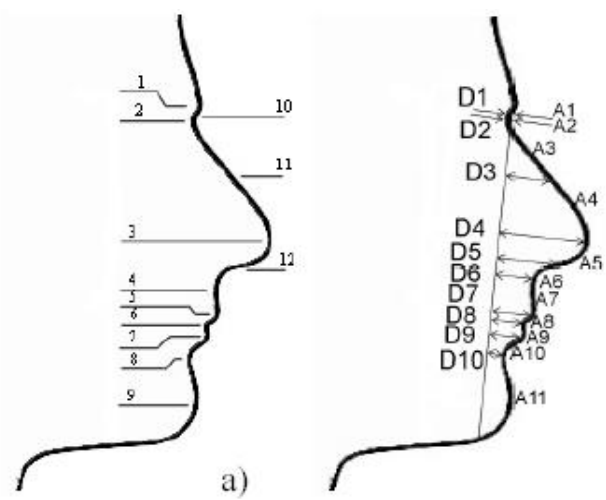

b)

Fig. 4 The twelve fiducial points of interest for face recognition; b) Feature vector has 21 components; ten distances D1-D10 and eleven profi A1-A11 [6]

\subsubsection{HOLISTIC APPROACH}

Holistic approaches attempt to identify faces using global representations, i.e., descriptions based on the entire image rather than on local features of the face.
These schemes can be subdivided into two groups: statistical and AI approaches. An overview of some of the methods in these categories follows.

\subsubsection{STATISTICAL APPROACH}

In the simplest version of the holistic approaches, the image is represented as a $2 \mathrm{D}$ array of intensity values and recognition is performed by direct correlation comparisons between the input face and all the other faces in the database. Though this approach has been shown to work under limited circumstances it is computationally very expensive and suffers from the usual shortcomings of straightforward correlation-based approaches, such as sensitivity to face orientation, size, variable lighting conditions, background clutter, and noise.

This sample of eigenfaces shows the tendency of the principal components to capture major variations in the training set such as lighting direction: [6]

\subsubsection{AI APPROACH}

AI approaches utilize tools such as neural networks and machine learning techniques to recognize faces. Some examples of methods belonging to this category are given below.

\subsubsection{HOW 3D PROCEDURE IS WORK [7]}

The use of depth and focus of the face that does not act the change in lighting is known as three-dimensional face recognition system. The software system that relay on three dimensional technique with a series of steps to eventually be able to perform a face recognition procedure. We can divide the whole process by the following steps.

\subsubsection{DETECTION:}

Capture a digital image by a two-dimensional digital camera or even using a video camera.

\subsubsection{ALIGNMENT:}

After capturing the image, the system will determine a head position, size and its direction. The threedimensional system can do this step even if the picture is diagonal taken. This will create an angle of 90 degrees with the camera lens, while the two dimensional system cannot perform this step only if the person is looking directly at the camera or in its direction as not to increase the angle between the person's face and camera lens of 35 degree.[7]

\subsubsection{MEASUREMENT:}

The software (specie program) will calculate the curves and meanders on the face to an accuracy of part OS the millimeter. Then the program ready to convert that information to establish a face model or pattern.

\subsubsection{REPRESENTATION:}

In this step, the system will translate the model and form a specie code. The code for each model is unique and consists of a set of numbers. 


\subsubsection{MATCHING:}

In the case that the picture is three-dimensional and corresponding to the three dimensional images that stored in the database, the comparisons between the images are immediately. But the challenge facing these systems is that most of the images stored in database are in twodimensional. So, how can be compared with a vivid picture of a person moves his head in front of camera and pick up his/here three-dimensional image with the millions of two-dimensional images the steps of 3D face recognition system .[7]

The development of a new technology support the use of three die rent points to get to know any face sorted in database. Some of these points are outside of the eyes, inside the eyes and the tip of the nose. The conduct of the system will carry out these measurements on the dimensions between these points of three-dimensional picture and begin to be converted to two-dimensional images through the application of complex mathematical algorithms. After the conversion process, of this part, the system begins to work of comparison. Verification or Identification: In the step of recognition, the program will compared the images and match them with pictures of the database sorted by the system in the previous step. But if the goal is verify the result of the previous step, the system compares the image with all images in the database and then matching results are displayed in percentages Feature Extraction Process.[7]

\subsubsection{NEW IDEA FOR RECOGNIZE THE FACE}

\subsubsection{FEATURE EXTRACTION}

For face recognition there are several steps as mention before used to recognize the person face. The step is to divide the human face into some region to reduce the search region for detect purpose. In this part we will show in more details how this procedure is done.

\subsubsection{FACE SEGMENTATION}

mentioned before the rst step in face recognition system is detecting the face and locate the face area from a given facial scan. The segmented face area starts from the forehead until the chin.. The subsequent feature point extraction is conducted within the segmented face area

\subsubsection{DRAWING (X, Y) AXES IN HUMAN FACE}

For drawing $(\mathrm{x}, \mathrm{y})$ axes in human face we have to set the center rst. A nose is a special point in human face and also holds the maximum height of the face. Sometimes other factors can affect the nose extract as beard, hair, other objects in the eld of view, sensor noise, and so on.

In $\mathrm{A}$ the beard will be detect as a maximum height and in $\mathrm{B}$ the hair.
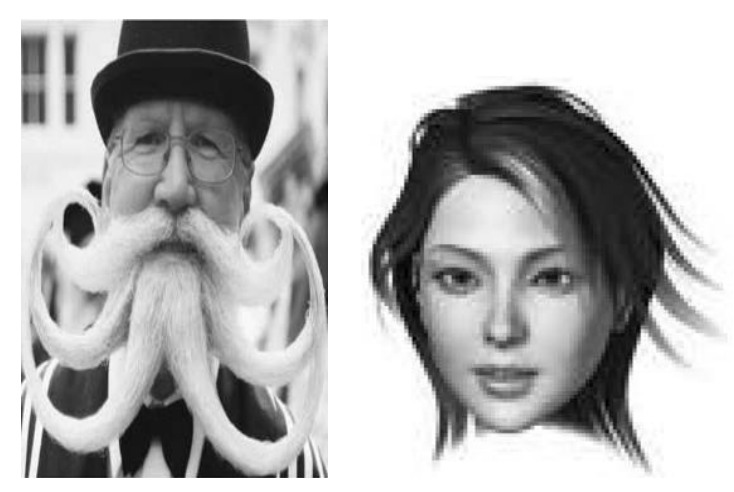

Fig. 6 Angle drawing [7]

For this problem we developed a robust nose tip extraction scheme. We look for the shape of the nose to locate it in the map. The range image is represented as $h(r$ $c)$, where $h$ is the height value, $r$ for the row indices and $c$ for the column indices. By using the face segmentation extraction as mentioned in part a, we will have the part of the person face only. Then we will the position of maximum $h$ by searching vertically and then draw the row in where $h$ is highest. After the row of the highest value of $h$ is being known and drawn, we will start searching horizontally and then draw the column in where $h$ is holding the maximum value. This process is shown in the picture. As you can see (x, y) axes have been drawn in the human face, and the nose is being in the centre.

\subsubsection{INFRA-RED}

Since thermal infra-red imagery of faces is relatively insensitive to variations in lighting, such images can hence be used as an option for detecting and recognizing faces. Furthermore, argues that since infra-red facial images reveal the vein and tissue structure of the face which is unique to each individual (like a fingerprint), some of the face recognition techniques for the visible spectrum should therefore yield favorable results when applied to these images.[6]

\section{DISCUSSION}

In recent years so many techniques are emerged for face recognition but still all are not $100 \%$ satisfactory, all the techniques have some advantages as well as some disadvantages

1. Linear Discriminant Analysis- This technique is extensively explored the illumination change and synthesis for facial analysis using appearance-based approaches to achieve an illumination-invariant but Not works properly under variations in pose and different lighting conditions. 
2. Viola and Jones, Classification and AdaBoost learning- These techniques are capable for face detection which minimizes computation time while achieving high detection accuracy but unlikely to be brittle or limited to a single set of conditions.

3. Curvature analysis- This method has Less false positives occurred, Effective in variant light conditions but artifacts introduced in acquisition.

4. Feature Extraction- This method helps the camera to do better auto-focusing and auto-exposure but not giving any idea for visual object detection.

\section{CONCLUSION}

In recent years face recognition has received substantial attention from researchers in biometrics, pattern recognition, and computer vision communities. There is a large number of commercial, security, and forensic applications requiring the use of face recognition technologies. As you can see, face recognition system is very important in our daily life. It is possesses a really great advantage. Among the whole types of biometric, face recognition system is the most accurate. In this paper the classification of face detection techniques been done with that some of the face recognition algorithms and techniques been discussed along with their advantage and disadvantage in tabular form.

\section{REFERENCES}

[1] Al-atrash, Shady S. "Robust Face Recognition." (2011).

[2] De Carrera, Proyecto Fin. "Face Recognition Algorithms." (2010).

[3] Wilson, Phillip Ian, and John Fernandez. "Facial feature detection using Haar classifiers." Journal of Computing Sciences in Colleges 21.4 (2006): 127-133.

[4] Scheenstra, Alize, Arnout Ruifrok, and Remco C. Veltkamp. "A survey of 3D face recognition methods." Audio-and Video-Based Biometric Person Authentication. Springer Berlin Heidelberg, (2005).

[5] Viola, Paul, and Michael J. Jones. "Robust real-time face detection." International journal of computer vision 57.2 (2004): 137-154.

[6] Jafri, Rabia, and Hamid R. Arabnia. "A Survey of Face Recognition Techniques." JIPS 5.2 (2009): 41-68.

[7] Al-Ghamdi, Bayan Ali Saad, Sumayyah Redhwan Allaam, and Safeeullah Soomro. "Recognition of Human Face by Face Recognition System using 3D." Journal of Information \& Communication Technology Vol 4: 27-34.

[8] Siddharth Swarup Rautaray and Anupam Agrawal, "Real Time Multiple Hand Gesture Recognition System for Human Computer Interaction", In International Journal of Intelligent Systems and Applications, 2012, 5, 56-64, DOI: 10.5815/ijisa.2012.05.08

[9] Song, Fengxi, et al. "A multiple maximum scatter difference discriminant criterion for facial feature extraction." Systems, Man, and Cybernetics, Part B: Cybernetics, IEEE Transactions on 37.6 (2007): 15991606.
[10] Bowyer, Kevin W., Kyong Chang, and Patrick Flynn. "A survey of approaches and challenges in 3D and multimodal 3D+2D face recognition." Computer Vision and Image Understanding 101.1 (2006): 1-15.

[11] Lu, Xiaoguang. "Image analysis for face recognition." personal notes, May 5 (2003).

[12] Zhang, Cha, and Zhengyou Zhang. A survey of recent advances in face detection. Tech. rep., Microsoft Research, (2010).

[13] Zhao, Wenyi, et al. "Face recognition: A literature survey." Acm Computing Surveys (CSUR) 35.4 (2003): 399-458.

[14] Abate, Andrea F., et al. "2D and 3D face recognition: A survey." Pattern Recognition Letters 28.14 (2007): 18851906.

[15] Colombo, Alessandro, Claudio Cusano, and Raimondo Schettini. "3D face detection using curvature analysis." Pattern recognition 39.3 (2006): 444-455.

[16] Zhou, Xuebing, et al. "A 3d face recognition algorithm using histogram-based features." Proceedings of the 1st Eurographics conference on $3 D$ Object Retrieval. Eurographics Association, 2008.

[17] Sadi, Vural. "Face recognition by using hybrid-holistic methods for outdoor surveillance systems." (2012).

[18] Belhumeur, Peter N. "Ongoing Challenges in Face Recognition." Frontiers of Engineering: Papers on Leading-Edge Engineering from the 2005 Symposium. 2005.

[19] Nigam, Aditya. A Novel Face Recognition Approach using Normalized Unmatched Points Measure. Diss. INDIAN INSTITUTE OF TECHNOLOGY, 2009.

[20] Vully, Mahesh Kumar. Facial expression detection using principal component analysis. Diss. 2011.

[21] Fladsrud, Tom, and False Acceptance Rate. "Face Recognition in a border control environment." Gjфvik University College (2005).

[22] Patra, Arpita. "Development of efficient methods for face recognition and multimodal biometry." (2006).

[23] Vuçini, Erald, Muhittin Gökmen, and Eduard Gröller. "Face recognition under varying illumination." Proceedings WSCG. 2007.

[24] Kumar, Pramod, Mrs Monika Agarwal, and Miss Stuti Nagar. "A Survey on Face Recognition System-A Challenge."

[25] Rautaray, Siddharth S., and Anupam Agrawal. "Vision based hand gesture recognition for human computer interaction: a survey." Artificial Intelligence Review (2012): $1-54$.

\section{Authors' Profiles}

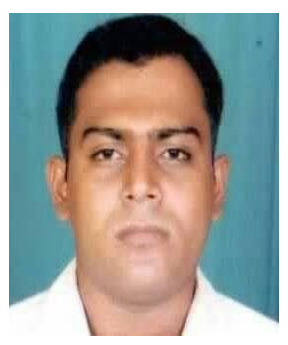

Mr.Subrat kumar Rath is presently pursuing m.tech in database engineering in School of Computer Engineering, KIIT University, Bhubaneswar, Odisha. His current research interest includes Image processing, Data mining, Cloud computing, no sql database. 


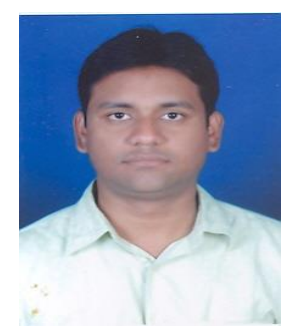

Siddharth S. Rautaray is presently working as an Assistant Professor in School of Computer Engineering, KIIT University, Bhubaneswar, Odisha. He is pursuing his Ph.D. in the Department of Information Technology at Indian Institute of Information Technology, Allahabad, India

His current research interests include human-computer interactions, user interface design and computer vision image processing. He has published around 17 research papers in different international and national journals and conferences. He is a member of the IEEE Computer Society, ACM SIGCHI and CSI.

How to cite this paper: Subrat Kumar Rath, Siddharth Swarup Rautaray,"A Survey on Face Detection and Recognition Techniques in Different Application Domain", IJMECS, vol.6, no.8, pp.34-44, 2014.DOI: 10.5815/ijmecs.2014.08.05 